\title{
Fourier Analysis of Submillimeter-Wave Scattering from the Human Cornea
}

\author{
Faezeh Zarrinkhat*†, Joel Lamberg $^{\dagger}$, Aleksi Tamminen ${ }^{\dagger}$, Mariangela Baggio ${ }^{\dagger}$, Juha \\ Ala-Laurinaho $^{\dagger}$, Elsayed E. M. Khaled ${ }^{\ddagger \S}$, Juan Manuel Rius*, Jordi Romeu Robert*, Zachary Taylor ${ }^{\dagger}$ \\ * CommSensLab, Technical University of Catalonia/UPC, Barcelona, Spain, faezh.zarrinkhat@tsc.upc.edu \\ $\dagger$ Department of Electronics and Nanoengineering, Millilab, Aalto University, Espoo, Finland, aleksi.tamminen@aalto.fi \\ $\ddagger$ Department of Electrical Engineering, Assiut University, Assiut, Egypt, esamk54_2000@aun.edu.eg \\ $\S$ Dean, High Institute of Engineering and Technology, Sohage, Egypt
}

\begin{abstract}
Wave scattering from a human cornea illuminated with a submillimeter-wave Gaussian beam is explored with Fourier analysis. This new approach enabled us to investigate the cornea as a coated sphere rather than a homogenized one. The cornea was modeled as an aqueous spherical shell using effective medium theory, with 60 percent water, enclosing a sphere of pure water. The corneal model was illuminated at $220 \mathrm{GHz}-330 \mathrm{GHz}$. The interaction of the incident and back-reflected beam, backscattered field, and back-scattering from one usual beam-cornea alignment scheme were evaluated; beam waist collocated with the surface apex. The result indicates the amount of difference between the reflection from planar stratified and back-scattering from the cornea in the case of focusing the beam waist at the corneal apex.
\end{abstract}

Index Terms-Cornea, Fourier analysis, sub-millimeter waves, vector spherical harmonics.

\section{INTRODUCTION}

At sub-millimeter wavelengths, the human cornea can be approximated as a lossy spherical shell of tissue encapsulating a sphere of water [1]. Locally, this structure reduces to a lossy thin film bordered by a half-space of air and half-space of water [2]. The layered structure dimensions suggest that the longitudinal modes exist in submillimeter wavelengths and enable extraction of corneal tissue permittivity and thickness leading to accurate quantification of tissue water content [3]. However, the curved structure presents challenges to ensuring that scattered phase fronts from primary, secondary, tertiary, etc. reflections are spatially coherent; a necessary condition for observing longitudinal modes.

Sub-millimeter wave and $\mathrm{THz}$ spectroscopy of cornea typically employs a lens or mirror to focus free space radiation onto the surface of corneal tissue or corneal phantoms [4]. Published illumination schemes can be broadly partitioned into two categories describing the Gaussian beam waist with respect to corneal geometry

- The Gaussian beam waist is positioned coincident with the corneal tissue/phantom apex

- The center of curvature of the corneal tissue/phantom is placed coincident with the focal point of the lens/mirror creating the focused Gaussian beam

In both cases, the scattered field is mapped to a complex reflection coefficient and the data fit to a plane wave on plane stratified media model. While this approach has produced good fits and permittivity commensurate with tissue/phantom water content, the applicability of planar stratified media models to media composed of concentric spherical shells has not been studied rigorously for cornea.

Our recent work in corneal water content quantification support the $W R-3.4$ band $(220 \mathrm{GHz}-330 \mathrm{GHz})$ as a suitable candidate for corneal water content sensing as the tissue loss is still low enough to support measurable longitudinal modes. However, the typical corneal RoC, normalized to midband $(275 \mathrm{GHz} \rightarrow 1.09 \mathrm{~mm})$ is $\sim 7.15 \lambda$ which makes broad band phase front matching difficult and further calls into question the applicability of the plane wave-based data analysis approach [5].

To address this uncertainty, the broadband scattering of the cornea from the case of the collocation of the beam waist on the apex is investigated by Fourier analysis. The advantage of this method compare to the previously used method; physical optic; is the possibility of probing the cornea as a multilayered spherical model instead of a homogenized one. The methodology is outlined in section II. In section III, the interaction of the incident and scattered field, the backscattered field, and the magnitude of coupling efficiency for the case of focusing waist radius on the apex are computed and depicted. Eventually, the summary and outcome can be found in section IV.

\section{ANALYSis OF GAUSSIAN BEAM ILLUMINATION ON A COATED SPHERE}

The calculation of the scattered field from a coated sphere, when illuminated by a Gaussian beam from an optical laser, is presented in [6]. The given approach is made up of three steps. First, the Gaussian beam is expanded as a summation of plane waves employing Fourier analysis. Second, each plane wave is written in terms of vector spherical harmonics (VSH) to apply it to the spherical boundary. Finally, the Gaussian beam scattering coefficients are computed by $\mathrm{T}$-matrix requiring scattering coefficients of a layered sphere when illuminated by a plane wave. Each step is briefly described in the following sub-sections. 
1) Modeling the Gaussian beam with summation of plane waves:

It is assumed that the incident beam propagates in the $z$ direction and is polarized in the $x z$ plane with the suppressed time variation $e^{-i \omega t}[6]$ :

$$
\mathbf{E}^{i}=E_{x}^{i} \mathbf{i}_{x}+E_{z}^{i} \mathbf{i}_{z},
$$

where $\mathbf{i}_{x}$ and $\mathbf{i}_{z}$ are unit vectors in the $x$ and $z$ directions. Also, the $\mathbf{E}^{i}, E_{x}^{i}$, and $E_{z}^{i}$ are functions of $x, y$, and $z$. The linearly polarized Gaussian beam is defined in the way that $x$-component of the field possess Gaussian distribution which its beam waist $w_{0}$ located at $(0,0,0)$ :

$$
E_{x}^{i}=\frac{w_{0}}{w(z)} e^{-\frac{x^{2}+y^{2}}{w^{2}(z)}} e^{i k \frac{x^{2}+y^{2}}{2 R(z)}} e^{i\left(k z-\arctan \left(z / z_{g}\right)\right)} .
$$

The Rayleigh length, radius, and radius of curvature of the beam are $z_{g}, w(z)$, and $R(z)$, respectively. Here, wave vector is defined as $\mathbf{k}=k_{x} \mathbf{i}_{x}+k_{y} \mathbf{i}_{y}+k_{z} \mathbf{i}_{z}$ and $k=|\mathbf{k}|=\left[k_{x}^{2}+\right.$ $\left.k_{y}^{2}+k_{z}^{2}\right]^{1 / 2}=2 \pi / \lambda$ is the wave number at the wavelength $\lambda$. For any arbitrary $\mathbf{k}$, the angles $\theta_{x}, \theta_{y}, \theta$, and $\phi$ are defined in space as in Fig 1 and accordingly, the components of $\mathbf{k}$ are delineated as [6]:

$$
\begin{aligned}
& k_{x}=k \cos \theta_{x}=k \sin \theta \cos \phi \\
& k_{y}=k \cos \theta_{y}=k \sin \theta \sin \phi \\
& k_{z}=k \cos \theta .
\end{aligned}
$$

The $z$-component of the electric field is obtained according to the incident wave polarization and the relation $\mathbf{E}_{\mathbf{i}} \cdot \mathbf{k}=0$ :

$$
E_{z}^{i}(x, y, z)=-\left(\frac{k_{x}}{k_{z}}\right) E_{x}^{i}(x, y, z) .
$$

In order to represent the Gaussian beam as a summation of plane waves, diffraction theory is applied. It implies that if the complex field distribution is Fourier-analyzed across any plane (here $z=0$ ), the various spatial Fourier components can be identified as plane waves traveling in different directions

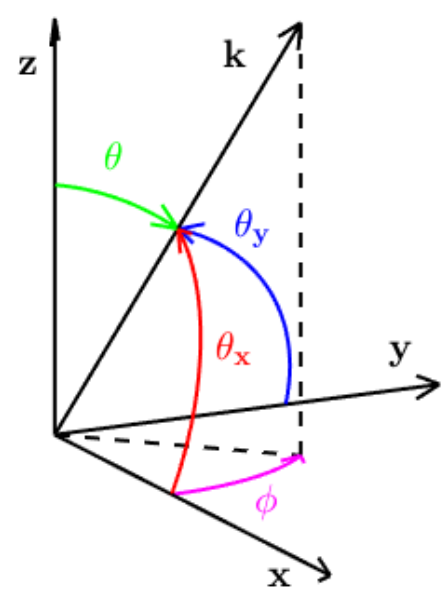

Fig. 1. Defining the propagation vector $\mathbf{k}$. The direction of $\mathbf{k}$ is defined by the $\theta$, and $\phi$ angles in the spherical coordinate system. The direction cosines angles are shown by $\theta_{x}, \theta_{y}$, and $\theta$. away from that plane. A two-dimensional Fourier transform of a Gaussian beam is another Gaussian beam with a different amplitude. The field amplitude and phase at any other point can be calculated by adding the contributions of these plane waves, taking account of the phase shifts $\left(e^{i k_{z} z}\right)$ they have undergone during propagation and then taking the inverse Fourier transform [7],

$$
E_{x}^{i}=C \iint e^{-\omega_{0}^{2} / 4\left(k_{x}^{2}+k_{y}^{2}\right)} e^{i\left(k_{x} x+k_{y} y+k_{z} z\right)} d k_{x} d k_{y},
$$

where coefficient $C=E_{0}^{i} \omega_{0}^{2} / 4 \pi$. To avoid evanescence waves, only real value of $k_{z}$ should be considered for integration. Total electric field $\mathbf{E}^{\mathbf{i}}$ is obtained according to (1), (4), and (5). Furthermore, (3) is used to change the variables for evaluating the integral as follows:

$$
\mathbf{E}_{\mathbf{i}}=C \iint e^{-\left(\frac{k \omega_{0} s}{2}\right)^{2}} e^{i k(\mathbf{s . r}-\mathbf{s . v})} \hat{e}_{x z} \sin \theta_{x} \sin \theta_{y} d \theta_{x} d \theta_{y},
$$

where $s^{2}=\cos ^{2} \theta_{x}+\cos ^{2} \theta_{y}$ and the vectors $\mathbf{s}, \mathbf{v}$, and $\mathbf{r}$ are $\mathbf{s}=\cos \theta_{x} \mathbf{i}_{x}+\cos \theta_{y} \mathbf{i}_{y}+\cos \theta \mathbf{i}_{z}, \mathbf{v}=x_{0} \mathbf{i}_{x}+y_{0} \mathbf{i}_{y}+z_{0} \mathbf{i}_{z}$, and $\mathbf{r}=x \mathbf{i}_{x}+y \mathbf{i}_{y}+z \mathbf{i}_{z}$, respectively. Also, the polarization factor $\hat{e}_{x z}=\left[\mathbf{i}_{x}-\cos \phi \tan \theta \mathbf{i}_{z}\right]$ and $\left(x_{0}, y_{0}, z_{0}\right)$ indicates beam waist location. In order to numerically calculate (6), rectangular rule technique is used. The final presentation will be:

$$
\mathbf{E}_{i} \approx F \sum_{\theta_{x}} \sum_{\theta_{y}} A_{\theta_{x y}}\left[\mathbf{i}_{x}-\frac{\cos \phi \sin \theta}{\cos \theta} \mathbf{i}_{z}\right] P W .
$$

In this representation, $F=\left(k \omega_{0} p\right)^{2} / 4 \pi$ in which $p=\pi / 180$ is the step size substituted $d \theta_{x}$ and $d \theta_{y}$. The $A_{\theta_{x y}}=$ $T G \sin \theta_{x} \sin \theta_{y}$ where $T=e^{-i k(\mathbf{s . v})}=e^{-i\left(k_{x} x_{0}+k_{y} y_{0}+k_{z} z_{0}\right)}$ indicates the relocation of waist radius relative to center of sphere and $G=e^{-\left(k s \omega_{0} / 2\right)^{2}}=e^{-\omega_{0}^{2} / 4\left(k_{x}^{2}+k_{y}^{2}\right)}$. In addition, $P W=e^{i k(\mathbf{s} . \mathbf{r})}=e^{i\left(k_{x} x+k_{y} y+k_{z} z\right)}$ illustrates a plane wave. In summary, (7) represents a Gaussian beam as a summation of plane waves.

\section{2) Expansion of Gaussian beam in terms of VSH:}

The VSH is the vector solution of the wave equation. It means it is possible to define any wave in space as a superposition of them. In the next step, the VSH expansion of the Gaussian beam is given according to the procedure described in [8]. Each plane wave at specific angles $\theta_{x}, \theta_{y}$, and $\theta$ can be written as a summation of the radial mode number $(n)$ and azimuthal modes $(m)$ :

$$
\mathbf{E}_{\theta_{\mathbf{x y}}}=\sum_{m} \sum_{n} D\left[a_{\theta_{x y}}^{e} \mathbf{M}_{e}^{1}+a_{\theta_{x y}}^{o} \mathbf{M}_{o}^{1}+b_{\theta_{x y}}^{e} \mathbf{N}_{e}^{1}+b_{\theta_{x y}}^{o} \mathbf{N}_{o}^{1}\right] \text {, }
$$

where $D=\frac{F \epsilon_{m}(2 n+1)(n-m) !}{4 n(n+1)(n+m) !}$ is a normalization factor along $\epsilon_{m}=1$ for $m=0$ and $\epsilon_{m}=2$ for $m>0$. The superscripts $e$ and $o$ denote even and odd modes. The $\mathbf{M}^{1}$ and $\mathbf{N}^{1}$ are the VSH of the first kind at any point in space [9]. Also, the 
expansion coefficients of each plane wave $a_{\theta_{x y}}^{e}, a_{\theta_{x y}}^{o}, b_{\theta_{x y}}^{e}$, and $b_{\theta_{x y}}^{o}$ yield as:

$$
\begin{aligned}
& a_{\theta_{x y}}^{e}=A_{\theta_{x y}}(\sin \phi \cos m \phi \tau-m \cos \phi \sin m \phi \Pi / \cos \theta) \\
& a_{\theta_{x y}}^{o}=A_{\theta_{x y}}(m \cos \phi \cos m \phi \Pi / \cos \theta+\sin \phi \sin m \phi \tau) \\
& b_{\theta_{x y}}^{e}=A_{\theta_{x y}}(m \sin \phi \sin m \phi \Pi+\cos \phi \cos m \phi \tau / \cos \theta) \\
& b_{\theta_{x y}}^{o}=A_{\theta_{x y}}(\cos \phi \sin m \phi \tau / \cos \theta-m \sin \phi \cos m \phi \Pi) .
\end{aligned}
$$

The $\Pi$ and $\tau$ are auxiliary functions given in [8]. The VSH expansion of incident Gaussian beam is a summation of plane waves as $\mathbf{E}^{\mathbf{i}}=\sum_{\theta_{x}} \sum_{\theta_{y}} \mathbf{E}_{\theta_{\mathbf{x y}}}$. Hence, the incident field presentation reads:

$$
\mathbf{E}^{\mathbf{i}}=\sum_{m} \sum_{n} D / F\left[a^{e} \mathbf{M}_{e}^{1}+a^{o} \mathbf{M}_{o}^{1}+b^{e} \mathbf{N}_{e}^{1}+b^{o} \mathbf{N}_{o}^{1}\right],
$$

where the incident field coefficients of the Gaussian beam are:

$$
\begin{aligned}
a^{e} & =F \sum_{\theta_{x}} \sum_{\theta_{y}} 4 i^{n} a_{\theta_{x y}}^{e}, \\
a^{o} & =F \sum_{\theta_{x}} \sum_{\theta_{y}} 4 i^{n} a_{\theta_{x y}}^{o}, \\
b^{e} & =F \sum_{\theta_{x}} \sum_{\theta_{y}}-4 i^{n+1} b_{\theta_{x y}}^{e}, \\
b^{o} & =F \sum_{\theta_{x}} \sum_{\theta_{y}}-4 i^{n+1} b_{\theta_{x y}}^{o} .
\end{aligned}
$$

\section{3) Scattering coefficient computed with T-matrix:}

The electric field scattered from a sphere illuminated with a Gaussian beam, $\mathbf{E}^{s}$, is obtained by combining the expansion coefficients from (11) with the T-matrix method which is a computational technique to compute scattering by arbitrary shape particles. The method is thoroughly explained in [8] Only the final formula is given here. The scattered electric field expansion is:

$$
\mathbf{E}^{s}=\sum_{m} \sum_{n} D / F\left[f^{e} \mathbf{M}_{e}^{3}+f^{o} \mathbf{M}_{o}^{3}+g^{e} \mathbf{N}_{e}^{3}+g^{o} \mathbf{N}_{o}^{3}\right],
$$

where the $\mathbf{M}^{3}$ and $\mathbf{N}^{3}$ are the VSH of the third kind and correspond to an outgoing wave [9]. The VSH expansion coefficients for the scattered electric field $f^{e}, f^{o}, g^{e}$, and $g^{o}$ from a sphere are obtained via the VSH expansion coefficients for the incident electric field as:

$$
\left[\begin{array}{l}
f^{e} \\
f^{o} \\
g^{e} \\
g^{o}
\end{array}\right]=-\left[\begin{array}{cccc}
T 11 & 0 & 0 & 0 \\
0 & T 22 & 0 & 0 \\
0 & 0 & T 33 & 0 \\
0 & 0 & 0 & T 44
\end{array}\right]\left[\begin{array}{l}
a^{e} \\
a^{o} \\
b^{e} \\
b^{o}
\end{array}\right] .
$$

$T$ matrix elements for the case of a coated sphere are related to $a_{n}^{c}$ and $b_{n}^{c}$, the scattering coefficients of a coated sphere when illuminated with a plane wave $[6,10]$ :

$$
\begin{aligned}
& T_{11}=T_{22}=-a_{n}^{c}, \\
& T_{33}=T_{44}=-b_{n}^{c} .
\end{aligned}
$$

The goal is to model the cornea with a radius of $R_{s}=7.5$ $\mathrm{mm}$. According to the operating frequency range for the imaging size parameter varies $34<x<52$. Suitable algorithm to calculate $a_{n}^{c}$ and $b_{n}^{c}$ for this size parameter is

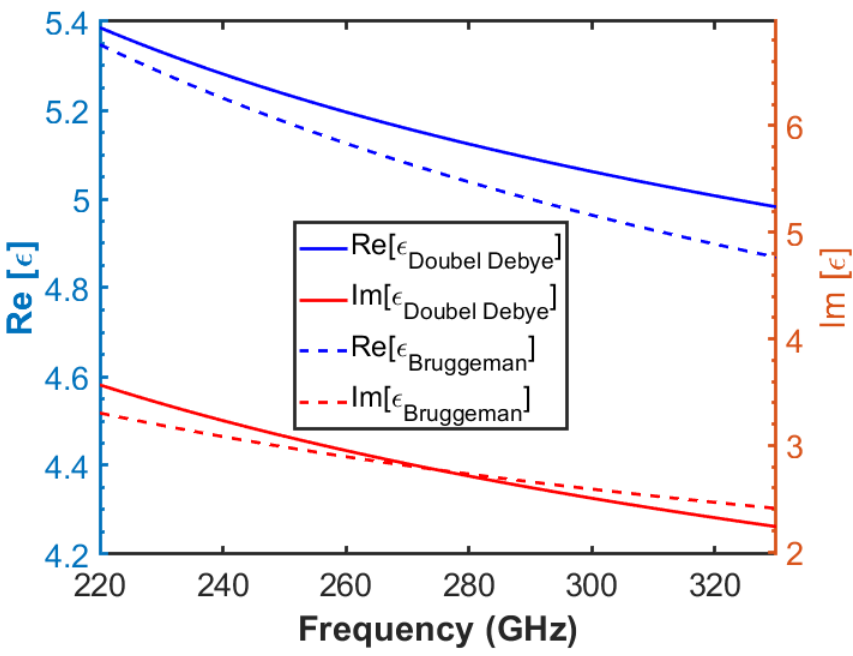

Fig. 2. Real (blue color) and imaginary (red color) part of the core (solid line) and shell (dotted line) permittivity are plotted as a function of the operating frequency. The water in core modeled with double-Debye model and the core (mixture of water and collagen) modeled with Bruggeman model

presented by Yang [11] and summarized by Peña in [12] as

$$
\begin{aligned}
a_{n}^{c} & =\frac{\left[H_{n}^{a}\left(\mathrm{~m}_{l} x_{l}\right) / \mathrm{m}_{l}+n / x_{l}\right] \psi_{n}\left(x_{l}\right)-\psi_{n-1}\left(x_{l}\right)}{\left[H_{n}^{a}\left(\mathrm{~m}_{l} x_{l}\right) / \mathrm{m}_{l}+n / x_{l}\right] \zeta_{n}\left(x_{l}\right)-\zeta_{n-1}\left(x_{l}\right)}, \\
b_{n}^{c} & =\frac{\left[H_{n}^{b}\left(\mathrm{~m}_{l} x_{l}\right) / \mathrm{m}_{l}+n / x_{l}\right] \psi_{n}\left(x_{l}\right)-\psi_{n-1}\left(x_{l}\right)}{\left[H_{n}^{b}\left(\mathrm{~m}_{l} x_{l}\right) / \mathrm{m}_{l}+n / x_{l}\right] \zeta_{n}\left(x_{l}\right)-\zeta_{n-1}\left(x_{l}\right)},
\end{aligned}
$$

where $\psi_{n}$ and $\zeta_{n}$ are Riccati-Bessel functions. The $\mathrm{m}_{l}$ and $x_{l}$ are refractive index and size parameter of the $l$ th layer. The $H_{n}^{a}$ and $H_{n}^{b}$ are given in [12].

\section{RESULTS}

As mentioned, the human cornea modeled with singlelayered sphere. The core and shell permittivity are set with the double-Debye and Bruggeman model, respectively as in

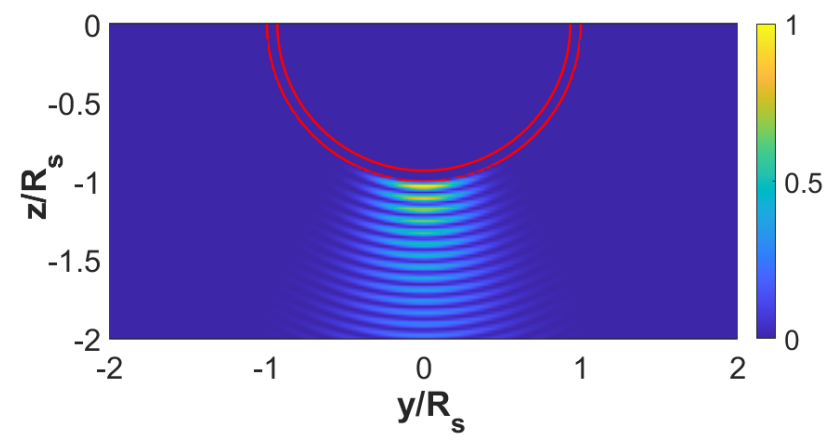

Fig. 3. Interaction of incident and back-scattered Gaussian beam in $y$ - $z$-plane The $y$ and $z$ axis are normalized with the radius of sphere $R_{s}$. The magnitude of the fields normalized with their maximum values. Each layer permittivity is set according to Fig. 2. 


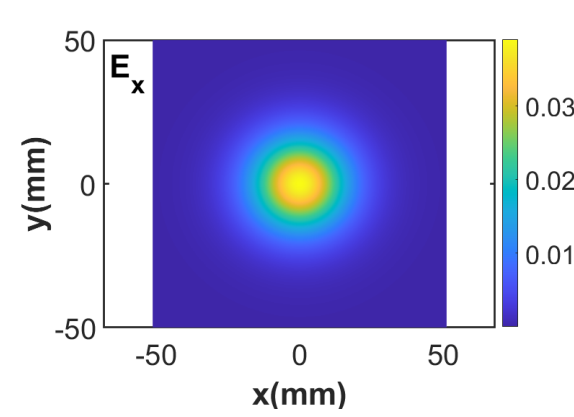

(a) $x$ component of back-scattered field

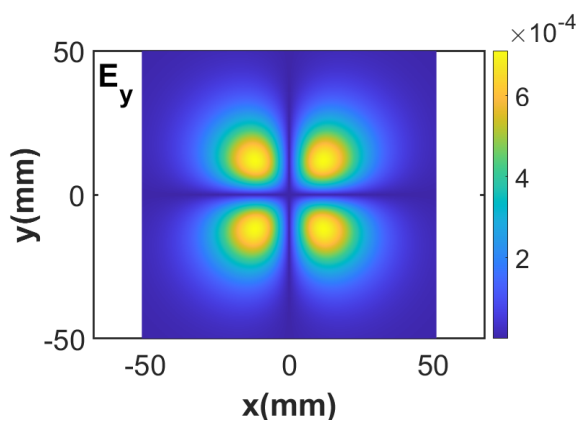

(b) $y$ component of back-scattered field

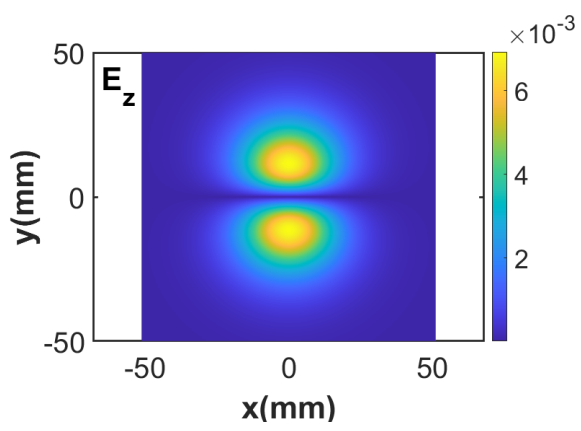

(c) $z$ component of back-scattered field

Fig. 4. The absolute scattered field from a one-layered sphere including core water and cornea shell is depicted in $x y$ plane at $z=-40 m m$ under the illumination of Gaussian beam polarized in $x$ and $z$ direction. The outer radius of coated sphere $R_{s}=7.5 \mathrm{~mm}$ and cornea (shell) thickness, thick $=500 \mu m$. The $x$ and $y$ axis are expanded from $-4 w(z)$ to $4 w(z)$. All the other parameters of the cornea and Gaussian beam are as in Fig. 3

Fig. 2. In Bruggeman model, free-water content is set to $60 \%$ and the solid-content permittivity is 2.9 . The shell thickness is $500 \mu \mathrm{m}$.

\section{A. Interaction of incident and back-scattered field}

The total field outside of the cornea equals to the vector summation of the incident and scattered beam (as in (10) and (12)), $\mathbf{E}=\mathbf{E}^{s}+\mathbf{E}^{i}$. It describes the interaction of the scattering and incident Gaussian beam which shows constructive and destructive interference between them.

In Fig. 3, $|\mathbf{E}|^{2}$ is plotted in $z$-y-plane. The $y$ and $z$ axis are normalized with the radius of the cornea $\left(R_{s}\right)$. Also, the $|\mathbf{E}|^{2}$ is normalized with respect to the maximum value. The operating frequency is $f=275 \mathrm{GHz}$, the beam focused on the center of the cornea and the beam waist is equal to the wavelength $\left(\omega_{0}=\lambda\right)$. To reach enough accuracy $N=30$ number of modes are considered. Also, to depict high resolution of the field interactions, 100 and 200 mesh grid points are taken into account in the $z$ and $y$ axis, respectively.

\section{B. The scattered field from cornea}

The absolute scattered field from the cornea at a plane parallel to $x$ - $y$-plane and at $z=-40 \mathrm{~mm}$ is calculated with (12) and illustrated in Fig 4. The value for $z$ is chosen according to the experimental setup where the radiating aperture locates at similar distance from the corneal apex [5]. In Fig 4a, 4b, and $4 \mathrm{c}$ scattered electric field components in $x, y$, and $z$ direction are depicted for $x$ and $y$ expanding from $-4 w(z)$ to $4 w(z)$ (considering 500 mesh grid points). In this calculation, beam waist is focused equal to the wavelength $w_{0}=\lambda$ (at mid-band frequency $f_{m}=275 \mathrm{GHz}$ ) and located at the center of the coordinate system $\left(x_{0}=0, y_{0}=0, z_{0}=0\right)$.

As mentioned, the incidence beam is polarized in $x$ and $z$ direction, the $z$-component is considered as a fraction of $x$-component, thereby $y$-component is zero. Likewise, the strongest amplitude of back-scattered field is $x$ component and $z$ component is stronger than $y$ component. Interestingly, the back-scattered field resembles a dipole and quadrupole behavior in $\mathrm{y}$ - and $\mathrm{z}$-direction. The dominant mode, related to $\mathrm{VSH}$, in $x, y$, and $z$ directions are $n=0, n=1$, and $n=2$, respectively.

\section{Coupling efficiency magnitude}

To give physical meaning to the calculation, coupling efficiency introduces as [13]:

$$
C E=\frac{\left|\iint \mathbf{E}^{i} \cdot \mathbf{E}^{s} d x d y\right|}{\iint\left|\mathbf{E}^{i}\right|^{2} d x d y} .
$$

It interprets as the magnitude of the coupling of the backreflected field to the incident field. For the case of focusing waist radius on the apex, the amplitude of coupling efficiency is computed at the reference plane at $z=-40 \mathrm{~mm}$ and shown in Fig. 5. In this scenario, the beam waist radius at the mid-band frequency focused on the middle of the shell. The waist radius location of the rest of the frequencies is set according to [5]. Also, Gaussian beam back-reflection and plane wave reflection coefficient from a plane boundary is plotted in Fig. 5. As shown in the inset figure, coupling efficiency of Gaussian beam illumination focused on the apex, deviates around $9-15 \%$ from plane wave illumination, which is roughly accurate but not enough, especially in lower frequencies. For better realization, the result is also shown for Gaussian beam illumination on a plane which is in good agreement with plane wave illumination on a plane surface (Fig. 5).

\section{CONCLUSION}

We have considered the scenario of focusing a Gaussian beam on the human cornea for corneal sensing at submillimeter waves. The scenario is modeled with a single-layered hydrated shell on an aqueous sphere. A comprehensive method based on Fourier analysis is used in the investigation. Also, an appropriate algorithm for the target size parameter is employed compared to the method presented earlier on coated spheres.

The interaction of incident and back-scattered field from the cornea is depicted. Also, the back-scattered field is computed and revealed an optimal alignment scenario for the Gaussian beam. On the other hand, by calculating the coupling efficiency, it is shown that focusing on the apex is not 

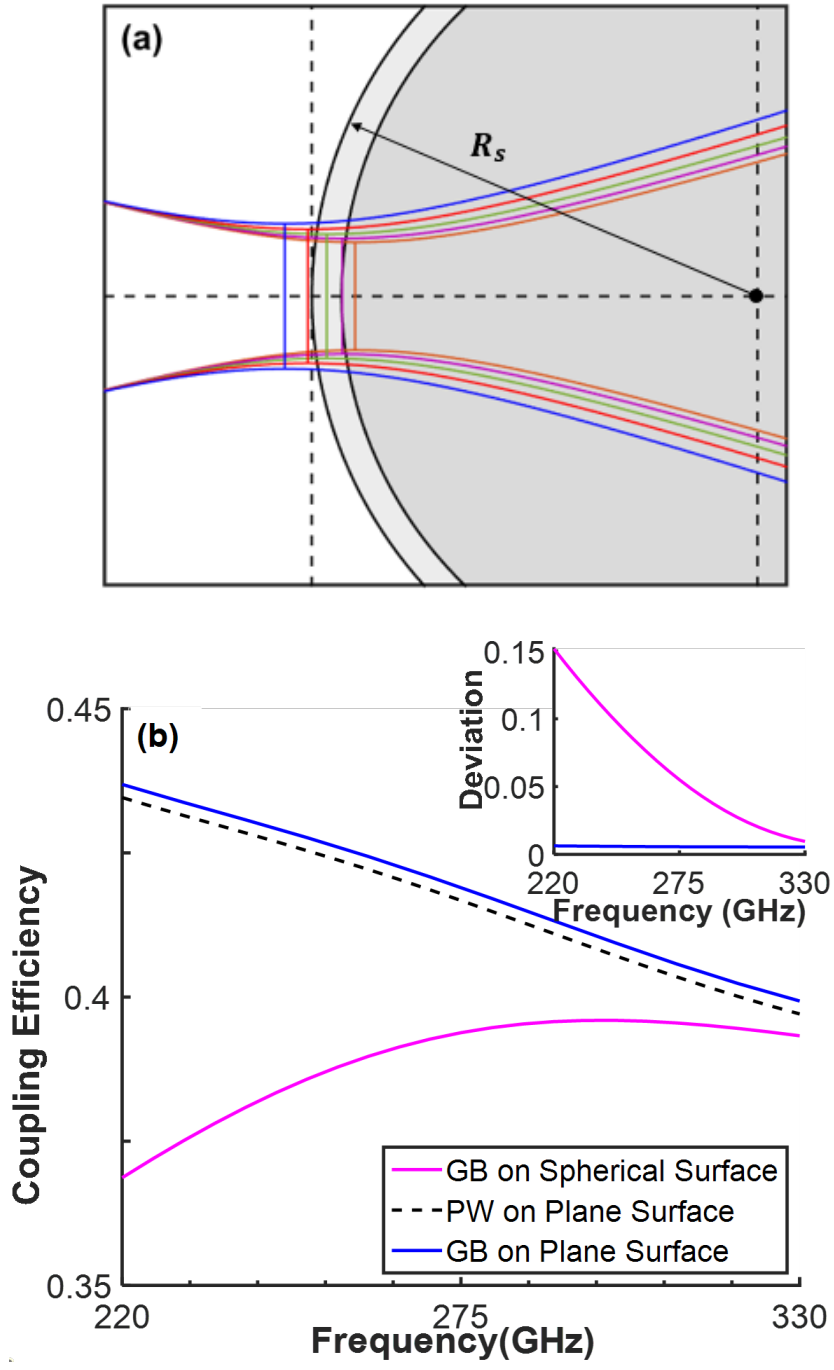

Fig. 5. (a) Scheme of focusing waist radius on the apex, (b) comparison of coupling efficiency amplitude as function of frequency. The inset shows the deviation of Gaussian beam illumination on sphere and plane from plane wave illumination on plane

relatively consistent with plane wave illumination on a plane and reveals a $9-15 \%$ relative deviation along with the operating frequency range- yet a scenario often occurring in the literature of corneal sensing. Hence, more investigation about proper focusing and phase investigation is required.

\section{REFERENCES}

[1] Z. D. Taylor, J. Garritano, S. Sung, N. Bajwa, D. B. Bennett, B. Nowroozi, P. Tewari, J. Sayre, J. P. Hubschman, S. Deng, E. R. Brown, and W. S. Grundfest, "THz and mm-wave sensing of corneal tissue water content: Electromagnetic modeling and analysis," IEEE Trans. Terahertz Sci. Technol., vol. 5, no. 2, pp. 170-183, 2015.

[2] Z. D. Taylor, J. Garritano, S. Sung, N. Bajwa, D. B. Bennett, B. Nowroozi, P. Tewari, J. W. Sayre, J. Hubschman, S. X. Deng, E. R. Brown, and W. S. Grundfest, "Thz and mm-wave sensing of corneal tissue water content: In vivo sensing and imaging results," IEEE Trans. Terahertz Sci. Technol., vol. 5, no. 2, pp. 184-196, 2015.

[3] S. Sung, S. Selvin, N. Bajwa, S. Chantra, B. Nowroozi, J. Garritano, J. Goell, A. D. Li, S. X. Deng, E. R. Brown, W. S. Grundfest, and Z. D. Taylor, "Optical System Design for Noncontact, Normal Incidence, THz Imaging of in vivo Human Cornea," IEEE Trans. Terahertz Sci. Technol., vol. 8, no. 1, pp. 27-37, 2018.

[4] _ , "Thz imaging system for in vivo human cornea," IEEE Trans. Terahertz Sci. Technol., vol. 8, no. 1, pp. 27-37, 2018.

[5] A. Tamminen, S. V. Pälli, J. Ala-Laurinaho, M. Salkola, A. V. Räisänen, , and Z. D. Taylor, "Quasioptics for corneal sensing at 220 - $330 \mathrm{GHz}$ : design, evaluation, and ex-vivo cornea parameter extraction," IEEE Trans. Terahertz Sci. Technol., 2020.

[6] E. E. M. Khaled, S. C. Hill, and P. W. Barber, "Scattered and internal intensity of a sphere illuminated with a gaussian beam," IEEE Transactions on Antennas and Propagation, vol. 41, no. 3, pp. 295-303, 1993.

[7] J. W. Goodman, Introduction to Fourier optics. Englewood, CO: Roberts, 2005.

[8] P. W. Barber and S. C. Hill, Light Scattering By Particles: Computational Methods. Advanced Series in Applied Physics, 1990.

[9] C. Bohren and D. R. Huffman, Absorption and Scattering of Light by Small Particles. Wiley Science Paperback Series, 1998.

[10] E. E. M. Khaled, S. C. Hill, and P. W. Barber, "Light scattering by a coated sphere illuminated with a gaussian beam," Appl. Opt., vol. 33, no. 15, pp. 3308-3314, 1994.

[11] W. Yang, "Improved recursive algorithm for light scattering by a multilayered sphere," Appl. Opt., vol. 42, no. 9, pp. 1710-1720, 2003.

[12] U. P. O. Peña, "Scattering of electromagnetic radiation by a multilayered sphere," IEEE Antennas Propag. Mag., vol. 57, no. 6, pp. 69-116, 2015.

[13] S. J. Orfanidis, Electromagnetic waves and antennas. Rutgers University New Brunswick, NJ, 2002. 